\title{
Selective Molecular Recognition of Arginine by Anionic Salt Bridge Formation with Bis-Phosphate Crown Ethers: Implications for Gas Phase Peptide Acidity from Adduct Dissociation
}

\author{
Ryan R. Julian* and J. L. Beauchamp \\ Beckman Institute, California Institute of Technology, Pasadena, California, USA
}

\begin{abstract}
Arginine forms a stable noncovalent anionic salt bridge complex with DP (a crown ether which contains two endocyclic dialkylhydrogenphosphate esters). Abundant adduct formation with DP is observed for complexes with arginine, YAKR, HPPGFSPFR, AAKRKAA, RR, RPPGFSPFR, RYLGYL, RGDS, and YGGFMRGL in electrospray ionization mass spectrometry (ESI-MS) experiments. DFT calculations predict a hydrogen bonded salt bridge structure with a protonated guanidinium flanked by two deprotonated phosphates to be the lowest energy structure. Dissociation of DP/peptide adducts reveals that, in general, the relative gas phase acidity of a peptide is dependent on peptide length, with longer peptides being more acidic. In particular, peptides that are six residues or more in length can stabilize the deprotonated C-terminus by extensive hydrogen bonding with the peptide backbone. Dissociation of $\mathrm{DP} /$ peptide complexes often yields the deprotonated peptide, allowing for the facile formation of anionic peptides that otherwise would be difficult to generate in high abundance. Although DP has a preference for binding to arginine residues in peptides, DP is also observed to form less abundant complexes with peptides containing multiple lysines. Lys-Xxx-Lys and Lys-Lys sequences form low abundance anionic adducts with DP. For example, KKKK exclusively forms a double adduct with one net negative charge on the complex. (J Am Soc Mass Spectrom 2004, 15, 616-624) (C 2004 American Society for Mass Spectrometry
\end{abstract}

$\mathrm{T}$ The development of methods that enable the rapid identification and characterization of proteins, especially those providing information relating to sequence, structure, and function, will facilitate new discoveries in the emerging field of proteomics [1, 2, 3]. Toward this goal, we have undertaken the development and systematic study of reagents that will selectively bind to peptides and proteins though specific noncovalent complexation of targeted amino acids both in solution and in the gas phase. For example, electrospray ionization (ESI) experiments employing 18-crown-6 ether (18C6) to form noncovalent complexes with lysine containing peptides and proteins have yielded valuable information about sequence and structure [4]. In these experiments, attachment of $18 \mathrm{C} 6$ to peptides yields quantitative information about the number of lysines present, whereas complexation with proteins reveals information about the tertiary structure and surface

Published online February 5, 2004

Address reprint requests to Dr. R. R. Julian, Department of Chemistry, Indiana University, 900 E. Kirkwood Avenue, Bloomington, IN 47504, USA. E-mail: ryjulian@indiana.edu

${ }^{*}$ Current address: Department of Chemistry, Indiana University, Bloomington, IN. availability of lysine residues [4]. In a related work, 18C6 serves as a molecular scaffold for the attachment of a diazo functional group, yielding a reagent known as a "molecular mousetrap" because of its ability to convert noncovalent complexes into covalently bound molecules following appropriate activation to form a reactive carbene [5].

Similar recognition of the side chain of arginine has been accomplished with larger crown ethers such as dibenzo-30-crown-10, which forms extensive hydrogen bonds with the protonated alkyl-guanidinium side chain of arginine [6]. Recognition of arginine can also be accomplished through the formation of salt bridges. A variety of solution phase reagents such as the arginine cork [7] which incorporates two carboxylates or Schrader and coworker's molecular tweezers [8] which contain two phosphates are based on the salt bridge approach. In the gas phase, Zenobi and co-workers have utilized disulfonic acids for the recognition of arginine in matrix assisted laser desorption ionization (MALDI) experiments [9]. Additionally, it has been shown recently that arginine residues can be monitored by covalent modification in solution followed by mass spectrometric analysis [10]. 


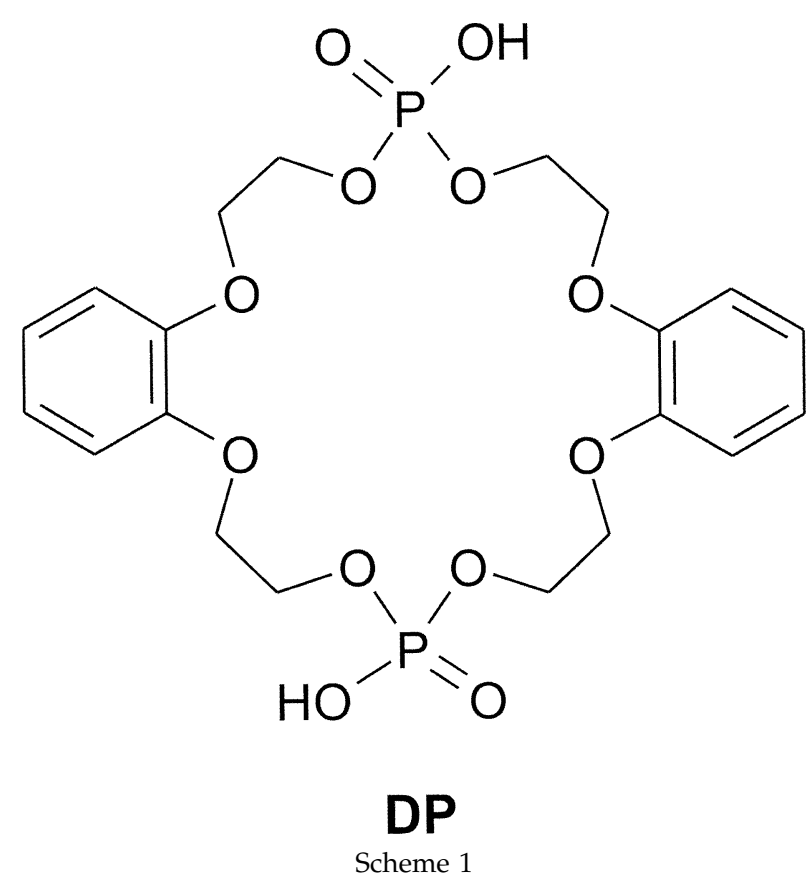

In the present work, the bis-phosphate crown ether DP [Dibenz(g,r) $(1,3,6,9,12,14,17,20,2,13)$ octaoxadiphosphacyclodocosin, 6,7,11,12,19,20,24,25-octahydro-9,22dihydroxy-, 9,22-dioxide (9CI)] is utilized for the selective recognition of the side chain of arginine employing ESI-MS to observe adduct formation. The structure and energetics of several key species and their adducts are characterized using theoretical calculations based on density functional theory (DFT). Theory predicts that the protonated alkyl guanidinium side chain of arginine forms a salt bridge with the two deprotonated phosphates incorporated into the crown ether. The noncovalent complexes that form are very abundant and easily detected by ESI-MS as anions. For this reason, we refer to DP as a charge changing complexation agent which enhances the selective detection of arginine containing peptides as anionic adducts. It is shown that dissociation of the anionic DP-peptide complexes often yields the deprotonated peptide, allowing for the facile formation of anionic peptides that otherwise would be difficult to generate in high abundance. Although DP does not yield anionic complexes with isolated lysine residues, it does form selective anionic salt bridge complexes with Lys-Xxx-Lys and Lys-Lys sequences in low abundance. The formation of nonselective adducts (in low abundance) between DP and various peptides is also investigated.

\section{Experimental Methodology}

All data were obtained using a Finnigan LCQ quadrupole ion trap mass spectrometer without modification. Soft sampling is crucial for the detection of these non-covalent complexes [11, 12]. The critical instrument settings that yield adduct formation include capillary voltage $14.12 \mathrm{~V}$, capillary temperature $200{ }^{\circ} \mathrm{C}$, and tube lens offset -39 V. Higher capillary temperatures can dissociate the crown ether/peptide complexes. The tube lens offset controls the acceleration of ions as they leave the capillary region. The tube lens voltage is minimized to reduce the number of energetic collisions with the He buffer gas. The upper mass limit for this instrument is $2000 \mathrm{~m} / \mathrm{z}$.

Sample concentrations were typically kept in the $\sim 10$ to $100 \mu \mathrm{M}$ range for all species of interest, unless otherwise noted. All samples were electrosprayed from a mixture of 80:20 methanol/water. Crown ethers were added in a 2- to 3-fold excess to the sample and electrosprayed with the analyte in order to observe adducts. Samples were electrosprayed with a flow of 3-5 $\mu \mathrm{L} / \mathrm{min}$ from a $500 \mu \mathrm{L}$ Hamilton syringe for optimal signal. Silica tubing with an inner diameter of 0.005 in. was used as the electrospray tip. No acid was added to any of the samples, unless otherwise noted. Identical solutions were used for the collection of both positive and negative ion ESI-MS. All chemicals unless otherwise noted were purchased from Sigma or Aldrich (St. Louis, MO) and used without further purification.

Where feasible, full geometry optimizations utilizing density functional theory were carried out at the B3LYP/6-31G** level using Jaguar 4.2 (Schrödinger, Inc., Portland, OR). Semi-empirical calculations were carried out using CACHe Worksystem Pro 5.04 (Fujitsu, Inc., Beaverton, OR). A variety of starting peptide structures were generated, then minimized to probe the conformational space of the system.

\section{Results and Discussion}

Before any experiments were performed, theory was used to quantitatively assess the structures and energetics of likely noncovalent complexes that could form between DP and the side chain of arginine. To conserve computational resources, propylguanidine was used as a model for arginine. DFT calculations at the B3LYP/6$31 G^{* *}$ level predict a salt bridge complex stabilized by favorable hydrogen bonding interactions between DP and propylguanidinium to be the lowest energy structure, as shown in Figure 1. Four hydrogen bonds and a nearly linear salt bridge stabilize this anionic noncovalent complex in the gas phase. Given the known affinity of crown ethers for cations in solution [13], and the fact that the interaction shown in Figure 1 can occur in solution as well as in the gas phase, it was predicted that DP would form abundant noncovalent complexes with arginine containing peptides that could be preserved and detected in the gas phase by experiments employing ESI-MS.

\section{Selective Complexation}

The ESI-MS results for complexing DP with arginine and the small peptide YAKR are shown in Figure 2. Arginine readily forms an abundant noncovalent com- 

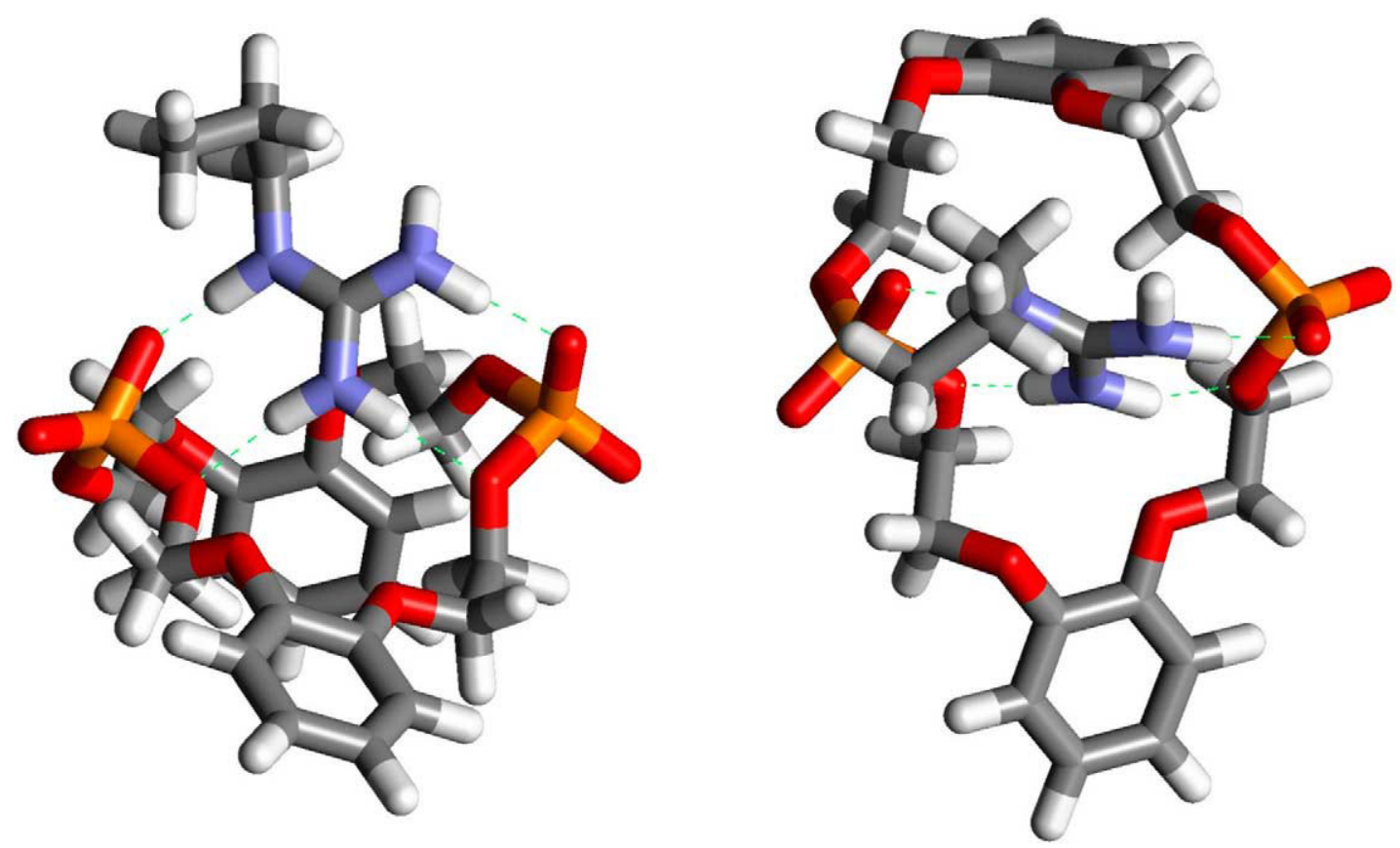

Figure 1. Two views of the lowest energy structure for the anionic adduct of propylguanidinium and DP. The structure was fully minimized at the B3LYP/6-31G** level and comprises a salt bridge with four strong hydrogen bonds between the central guanidinium cation and the two flanking phosphate anions.

plex with DP as shown in Figure 2a, confirming the predictions made based on the DFT calculations. The base peak in the spectrum corresponds to [DP + Arg $\mathrm{H}]^{-}$. Collisional activation of this peak leads primarily to the loss of neutral arginine as shown in Figure 2b. An additional minor loss of $87 \mathrm{Da}$ is observed. This loss must result from the cleavage of a covalent bond (several possible fragmentations of arginine lead to a loss of $87 \mathrm{Da}$ ), suggesting that the complex is formed with a reasonably high binding energy. Comparable experiments with lysine reveal no anionic adduct formation, suggesting that DP selectively attaches to arginine. Figure 2c illustrates complexation of DP with the small peptide $Y A K R$, which contains a single arginine. The complex [DP + YAKR $-\mathrm{H}]^{-}$is formed in high abundance. In fact, it is more abundant than the peak corresponding to deprotonated DP and is the most abundant anionic species derived from the peptide. By comparison, the $\left[Y A K R-\mathrm{H}^{-}\right.$peak is seven times less abundant. Collisional activation of the [DP + YAKR $\mathrm{H}]^{-}$complex in Figure 2d results predominantly in loss of the neutral peptide. There is no significant formation of a double adduct, where the second DP could theoretically attach to the lysine residue. The selectivity of DP for arginine over lysine stems from the much higher basicity of the guanidinium group. In solution, DP can form a salt bridge complex with both arginine and lysine, but upon transfer to the gas phase, a single lysine is insufficiently basic to stabilize the salt bridge interaction. Once in the gas phase, a proton transfer from the lysine to one of the phosphates on the DP would likely lead to dissociation of the DP from lysine.
Data for DP interacting with two larger peptides, YGGFMRGL and HPPGFSPFR, are shown in Figure 3. YGGFMRGL is an eight residue peptide with a single internal arginine. The results in Figure 3a show that this peptide forms an abundant anionic noncovalent complex with DP. In contrast to the results shown in Figure 2 , isolation of this complex followed by collisional activation leads almost exclusively to the loss of neutral DP as shown in Figure 3b. Figure $3 c$ shows the mass spectrum obtained from a solution of HPPGFSPFR and DP. This peptide is an analog of bradykinin with the $\mathrm{N}$-terminal arginine replaced with a histidine. It contains a single C-terminal arginine. The data in Figure $3 c$ shows an abundant noncovalent complex between the peptide and DP. Again, there is no significant formation of a double adduct, suggesting that histidine is not sufficiently basic to form a stable salt bridge with DP in the gas phase. Isolation of [HPPGFSPFR $+\mathrm{DP}-\mathrm{H}]^{-}$ followed by collisional activation leads primarily to the loss of neutral DP.

In Figure 4, the possibility of attaching multiple DP crowns to peptides containing multiple arginines is explored. Complexation with the dipeptide RR reveals that multiple attachments are possible as shown in Figure $4 \mathrm{a}$. The intensity of the double adduct [RR + 2DP $-\mathrm{H}]^{-}$is comparable to that for the single adduct $[\mathrm{RR}+\mathrm{DP}-\mathrm{H}]^{-}$. Note that both complexes are singly charged, suggesting that either the $\mathrm{N}$-terminus or one of the phosphates is protonated in the double adduct. A protonated N-terminus would likely result in an extended salt bridge structure, whereas protonation of the phosphate would lead to a neutral salt bridge between 

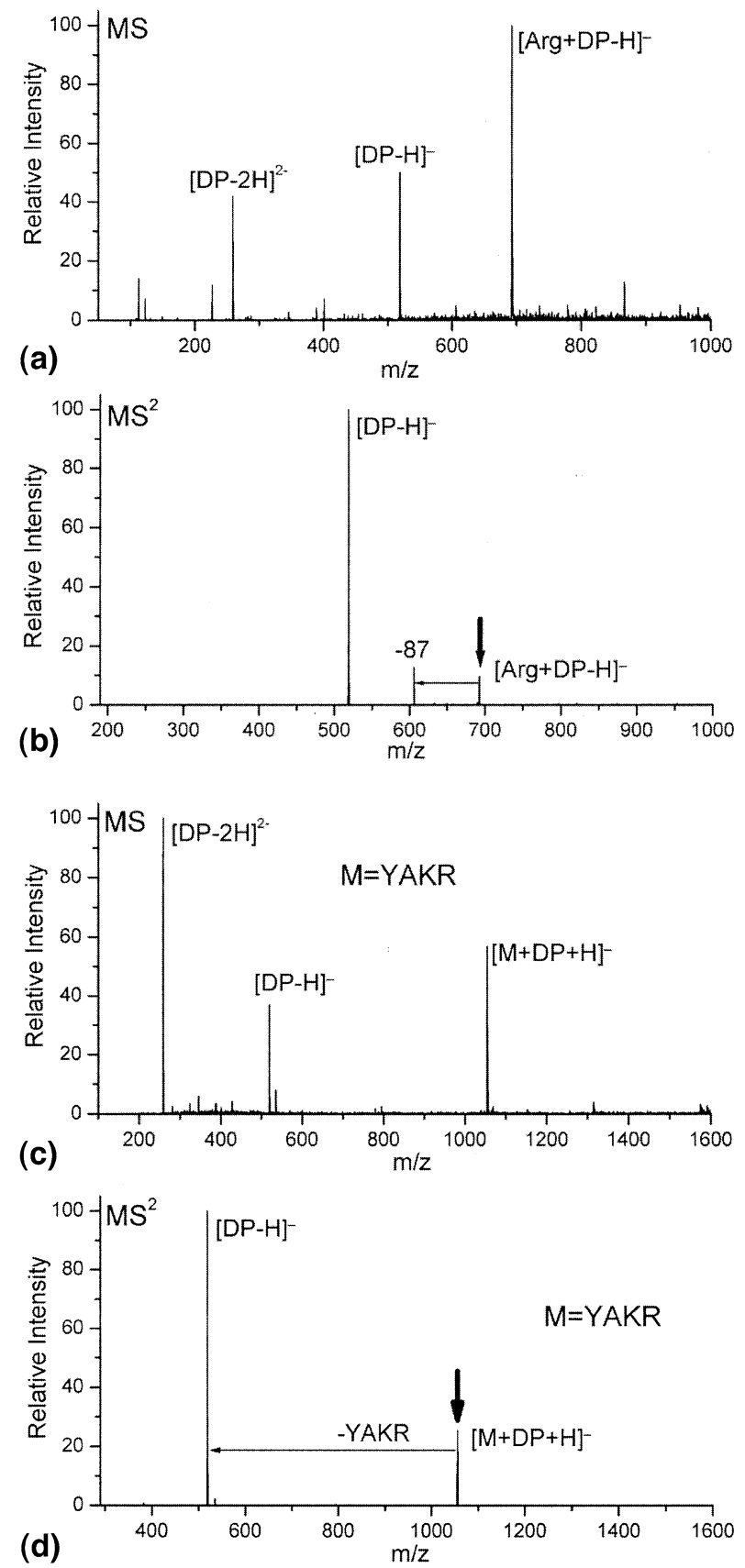

Figure 2. (a) Negative ion ESI-MS data for arginine with DP demonstrating abundant adduct formation. (b) Collisional activation of $[\mathrm{DP}+\mathrm{Arg}-\mathrm{H}]^{-}$yields loss of arginine. (c) Negative ion ESI-MS data for YAKR with DP, again demonstrating significant adduct formation. (d) $\mathrm{MS}^{2}$ spectra revealing loss of the neutral peptide. Bold downward arrows indicate peaks being subjected to collisional activation.

DP and one of the arginines. In Figure $4 \mathrm{~b}$, collisional activation of $[\mathrm{RR}+2 \mathrm{DP}-\mathrm{H}]^{-}$leads primarily to loss of neutral DP. In contrast, collisional activation of $[R R+$ $\mathrm{DP}-\mathrm{H}]^{-}$leads entirely to loss of neutral RR (data not shown). A dimer with two crowns and two peptides $\left([2 \mathrm{RR}+2 \mathrm{DP}-\mathrm{H}]^{-}\right)$is also present in low abundance. The doubly charged double adduct is not observed.

Bradykinin (RPPGFSPFR) is a larger peptide that
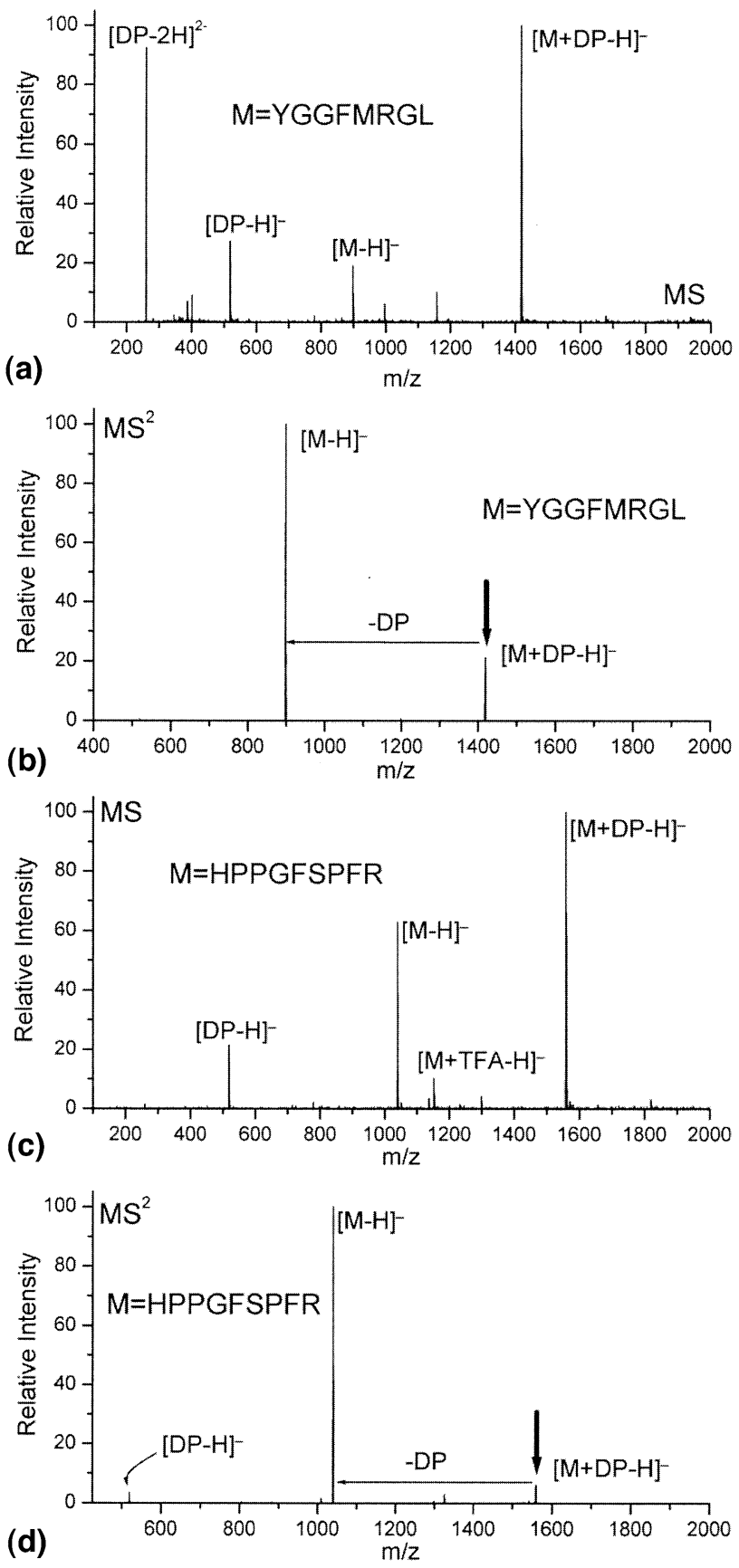

Figure 3. (a) Negative ion ESI-MS data for DP with YGGFMRGL yielding abundant adduct formation. (b) $\mathrm{MS}^{2}$ spectrum revealing loss of neutral DP. (c) Negative ion ESI-MS data for HPPGFSPFR with DP showing abundant complexation. (d) $\mathrm{MS}^{2}$ spectrum again revealing loss of neutral DP, suggesting that the peptide has the greater gas phase acidity. Bold downward arrows indicate peaks being subjected to collisional activation.

also contains two arginines. The mass spectrum obtained from a solution of bradykinin and DP is shown in Figure 4c. A single DP attaches in high abundance, but the singly charged double adduct is beyond the mass range of the instrument. A doubly charged double adduct is observed, but in very small abundance. There 

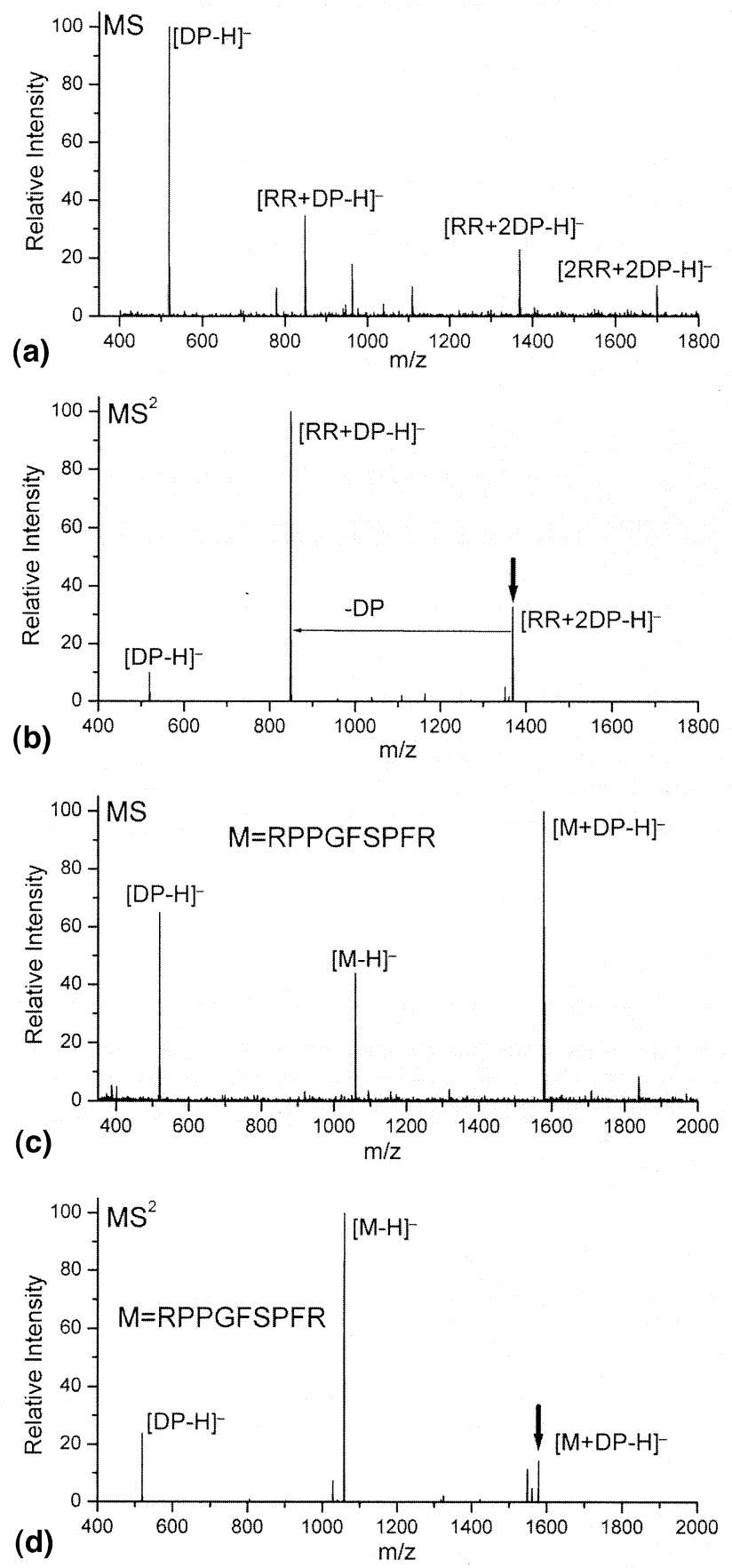

Figure 4. (a) Negative ion ESI-MS data for a mixture of DP and the dipeptide RR showing multiple adduct formation is possible. (b) Collisional activation leads to the loss of DP followed by the loss of RR. (c) Negative ion ESI-MS data for DP with bradykinin. The second adduct, if present, is beyond the mass range of the instrument. (d) Collisional activation leads primarily to the loss of DP. Bold downward arrows indicate peaks being subjected to collisional activation.

are several possible explanations for the low intensity of this peak. Sterically, the attachment of two DP crowns should be possible for a peptide the size of bradykinin, but the proximity of the two negative charges introduces coulombic repulsion on the order of $\sim 14 \mathrm{kcal} /$ mol, which may reduce the intensity of the double adduct. Alternatively, the low intensity of the double adduct may be a result of the compact conformation that bradykinin preferentially adopts in the gas phase. For example, singly protonated bradykinin forms a compact salt bridge structure in the gas phase [14]. Attachment of doubly deprotonated DP to this structure would lead to an anionic complex. However, if the intramolecular salt bridge that forms with the second arginine in the folded structure is energetically more favorable in the gas phase, the second adduct may be lost in favor of forming the intramolecular salt bridge. In fact, both of these factors may contribute to the low intensity of the doubly charged, double adduct. In Figure $4 d$, collisional activation results primarily in the neutral loss of DP. Some minor covalent fragmentation is also observed (loss of 17 and $30 \mathrm{Da}$ ).

\section{Nonselective Complexation}

For reasons that are not entirely understood, polyglycines also form reasonably abundant adduct peaks with DP. Figure 5 shows the results for two experiments with polyglycines that do not contain arginine, yet form weakly bound anionic noncovalent complexes. The abundance of adducts is lower than the intensity of the peak corresponding to the singly deprotonated DP, which was found to be an accurate indicator of peptides not containing arginine (at least for all of the larger peptides that we tested). In Figure $5 \mathrm{a}$, the spectrum obtained from a solution of hexaglycine and DP is shown. A small amount of complexation is observed. It is likely that this noncovalent complex forms because the absence of side chains allows for extensive hydrogen bond formation between the peptide backbone and DP. Interestingly, collisional activation yields primarily the deprotonated peptide. By way of comparison, under nearly identical conditions the observed total ion count for [GGGGGG $-\mathrm{H}]^{-}$on our instrument was $1.8 \times 10^{3}$ ions in the absence of DP, while the ion count for [GGGGGG + DP $-\mathrm{H}]^{-}$was $1.33 \times 10^{5}$ ions when DP was added. This represents an increase in signal of almost two orders of magnitude, and suggests a new method for generating abundant anionic peptides in the gas phase for any peptide that will complex with DP.

\section{Gas Phase Acidities}

The proton affinities of peptides are largely determined by the basicity of the side chains which are present within the peptide, with peptides containing multiple lysines or arginines being more basic than peptides with less basic side chains. Although it is very difficult to determine quantitative thermodynamic information for large molecules such as peptides, qualitative comparisons utilizing kinetic methods are possible. To our knowledge, no study on the gas phase acidity of entire peptides has been reported. Herein we report qualitative results comparing the gas phase acidity of several 

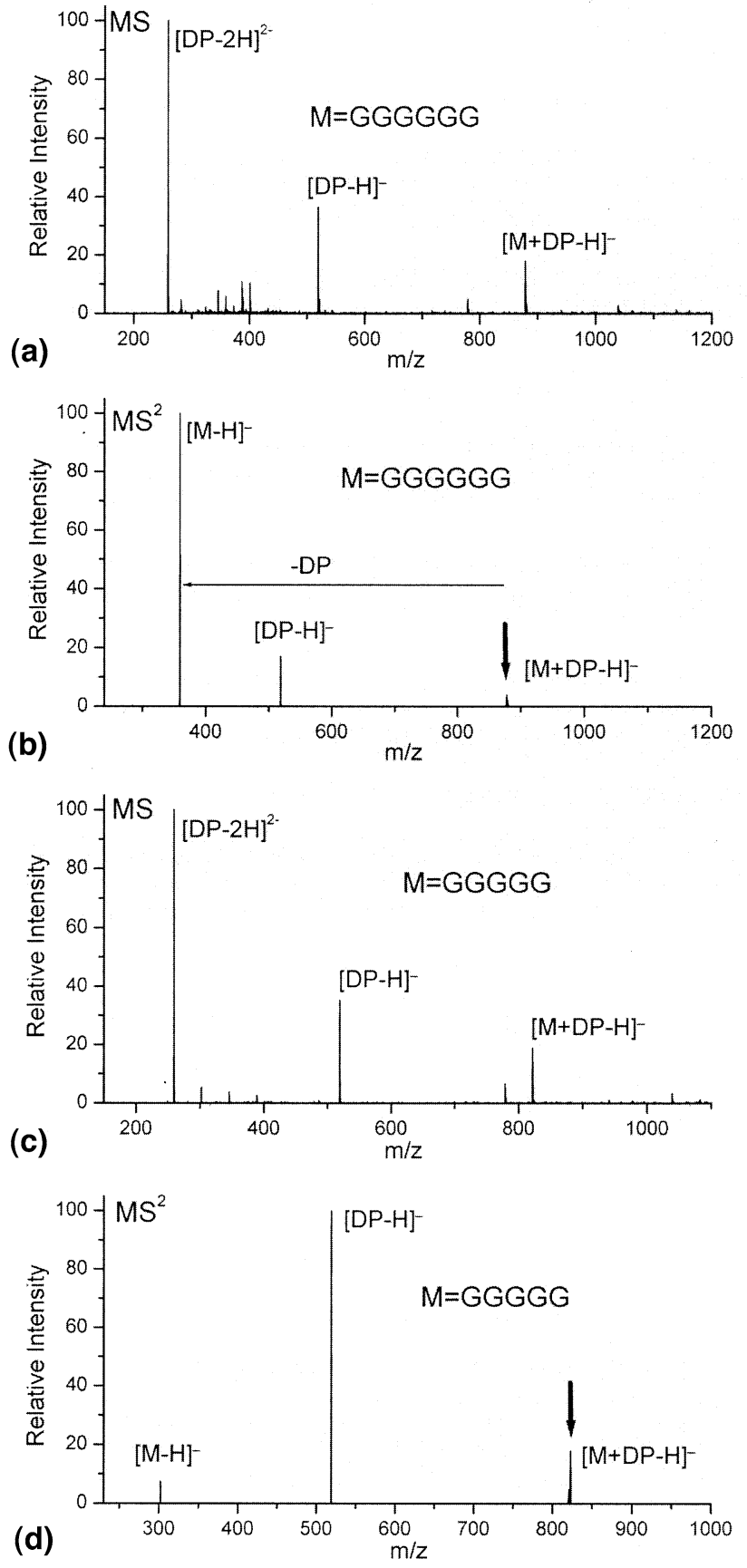

Figure 5. (a) Hexaglycine forms a nonspecific complex with DP in low abundance. (b) Collisional activation of the complex yields primarily deprotonated hexaglycine. (c) Pentaglycine also forms a nonspecific adduct with DP in low abundance. (d) In this case, collisional activation leads primarily to the loss of neutral peptide. Bold downward arrows indicate peaks being subjected to collisional activation.

peptides with that of DP. Collisional activation of [GGGGGG + DP $-\mathrm{H}^{-}$leads primarily to the loss of neutral DP, suggesting that hexaglycine is more acidic than DP. This result is counterintuitive because phosphates are generally more acidic than carboxylates. As seen in Figure 5c, pentaglycine also forms a nonspecific complex with DP in low abundance. However, upon
Table 1. Peptide Acidities Relative to DP

\begin{tabular}{lr}
\hline Less Acidic & More Acidic \\
\hline \hline Arg & YGGFMRGL \\
RR & HPPGFSPFR \\
RGDS & RPPGFSPFR \\
YAKR & AAKRKAA \\
GGGG & GGGGGG \\
GGGGG & RYLGYL \\
& DDDD \\
\hline
\end{tabular}

collisional activation of this peak, the neutral peptide is lost almost exclusively (Figure 5d). This suggests that pentaglycine is less acidic than DP, in contrast to hexaglycine. Similarly, DP is more acidic than tetraglycine. Upon dissociation of the noncovalent DP/peptide complex, the C-terminus is the most likely site for deprotonation for these oligoglycines which do not contain an acidic side chain. Based on this premise, it appears that the deprotonated C-terminus for hexaglycine receives additional stabilization relative to shorter oligoglycines, which effectively enhances the acidity of the longer peptide.

The acidities of the all of the peptides in the current study relative to DP are presented in Table 1 . The primary sequence of these peptides does not suggest an obvious approach for predicting acidity. For example, YAKR and AAKRKAA contain similar side chains but have opposite relative acidities. RGDS contains an acidic side chain, but it is less acidic than RYLGYL which contains no acidic side chains. Scrutiny of the data in Table 1 suggests that it is primarily the length of the peptide that plays the crucial role in determining acidity, with peptides that comprise six or more residues being more acidic than DP and shorter peptides being less acidic. In fact, this trend holds for all but one of the peptides studied in the present work. The only exception is in the case of tetraaspartic acid, where it is apparent that multiple acidic side chains can enhance the acidity of a peptide.

Calculations on deprotonated hexaglycine support the conjecture, hinted at by the experiments reported above, that solvation of the deprotonated C-terminus by the peptide backbone may enhance the acidity of longer peptides, independent of the side chains that are present (see Figure 6). Calculations at the PM5 semiempirical level of theory indicate that the total energy of deprotonated hexaglycine decreases by $23 \mathrm{kcal} / \mathrm{mol}$ when the extended structure is allowed to fold to form the optimized hydrogen bonded structure in Figure 6 . Therefore, the formation of three hydrogen bonds between the deprotonated C-terminus and the peptide backbone is energetically favorable. Since the lowest energy structure for anionic and neutral hexaglycine are both folded in a similar fashion, extensive hydrogen bonding with the peptide backbone is predicted to substantially increase the gas phase acidity of peptides with 6 or more residues in length. Although structurally similar to folded hexaglycine, for pentaglycine the 


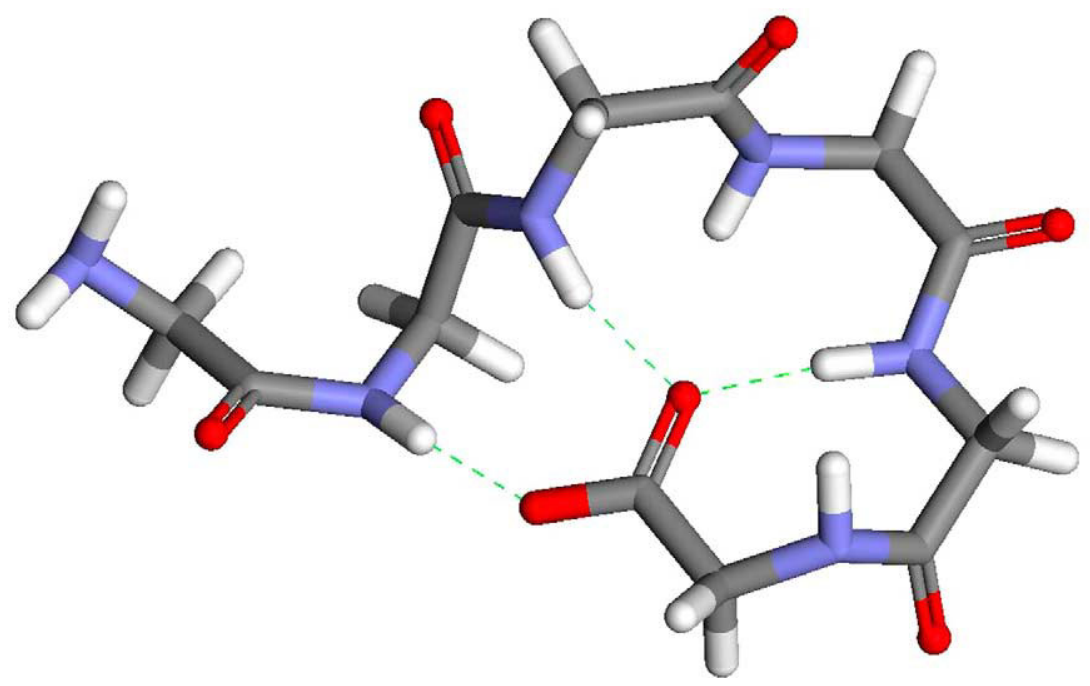

Figure 6. PM5 structure for deprotonated hexaglycine. Three hydrogen bonds with the peptide backbone stabilize the deprotonated C-terminus, effectively increasing the acidity.

C-terminus is only stabilized by $\sim 18 \mathrm{kcal} / \mathrm{mol}$ relative to the extended structure. This suggests that the gas phase acidity of DP lies within the $5 \mathrm{kcal} / \mathrm{mol}$ range separating hexaglycine and pentaglycine. The gas phase acidity of dimethyl phosphate is $325 \mathrm{kcal} / \mathrm{mol}$ [15]. Similarly, the gas phase acidity of arginine is 325 $\mathrm{kcal} / \mathrm{mol}[16]$, but the data in Figure $2 \mathrm{~b}$ suggests that DP is more acidic than arginine, implying that it has a gas phase acidity lower than $325 \mathrm{kcal} / \mathrm{mol}$. It is not our intent to quantify the acidity of the peptides in Table 1, but by way of qualitative comparison all of the peptides that are more acidic than DP have gas phase acidities lower than $325 \mathrm{kcal} / \mathrm{mol}$.

\section{Multiple Site Binding of DP to Small Peptides}

Figure 7a shows the results for adding DP to a solution of tetralysine. In this case, a selective attachment of two DP crowns to the peptide is observed, albeit in low abundance. The adducts in this case are not the result of non-selective complexation as was the case with hexaglycine. DP can form an extended salt bridge with Lys-Lys or Lys-Xxx-Lys sequences. The most favorable salt bridge that can be formed from this interaction is neutral, which may explain the low abundance of the anion observed in Figure 7a. Collisional activation of this double adduct reveals something interesting (Figure $7 \mathrm{~b}$ ). The primary initial loss is DP, followed by the loss of tetralysine. These losses are not unexpected. However, there is an additional peak corresponding to the initial loss of tetralysine, leaving behind a deprotonated DP dimer. This dimer peak is also generated by dissociation of the doubly charge double DP adduct with bradykinin. In the absence of other ligands, DP clusters readily when it is electrosprayed alone. Therefore, it appears that this predisposition for clustering leads to an alternate dissociation pathway producing a DP dimer. It is unlikely that the two DP crowns are dimerized prior to collisional activation because double adducts are not observed in every case. Nevertheless, it is impossible to eliminate this possibility within the confines of the present experiment.

The motifs recognized by DP can also be combined in a single peptide such as AAKRKAA, yielding multiple DP adducts as shown in Figure 7c. As expected, the single adduct is observed in high abundance, while the second adduct is present in much lower intensity. It should also be noted that collisional activation of [AAKRKAA + 2DP $-\mathrm{H}]^{-}$yields a DP dimer in small abundance similar to that observed in Figure $7 \mathrm{~b}$.

\section{Conclusion}

Molecular recognition of the side chain of arginine in small peptides is achieved by the selective and abundant formation of anionic noncovalent complexes with DP. The recognition results from the formation of four specific hydrogen bonds and an anionic salt bridge between the protonated guanidinium group of arginine and the deprotonated phosphates in DP. The complexes are formed with high specificity and abundance. In addition to attachment to arginine, multiple lysine residues also form less abundant noncovalent complexes with DP. Dissociation of the anionic complexes often yields the deprotonated peptide, suggesting a new method for the facile preparation of deprotonated peptides in the gas phase in high abundance. Furthermore, peptides with six or more non-proline residues are predicted to be more acidic than DP, due to extensive stabilization of the deprotonated C-terminus by the amide hydrogens of the peptide backbone. Complexation of DP with multiple arginines is also possible, but abundant multiply charged anionic complexes are not observed in peptides or proteins containing multiple arginine residues.

The above observations may have some interesting 

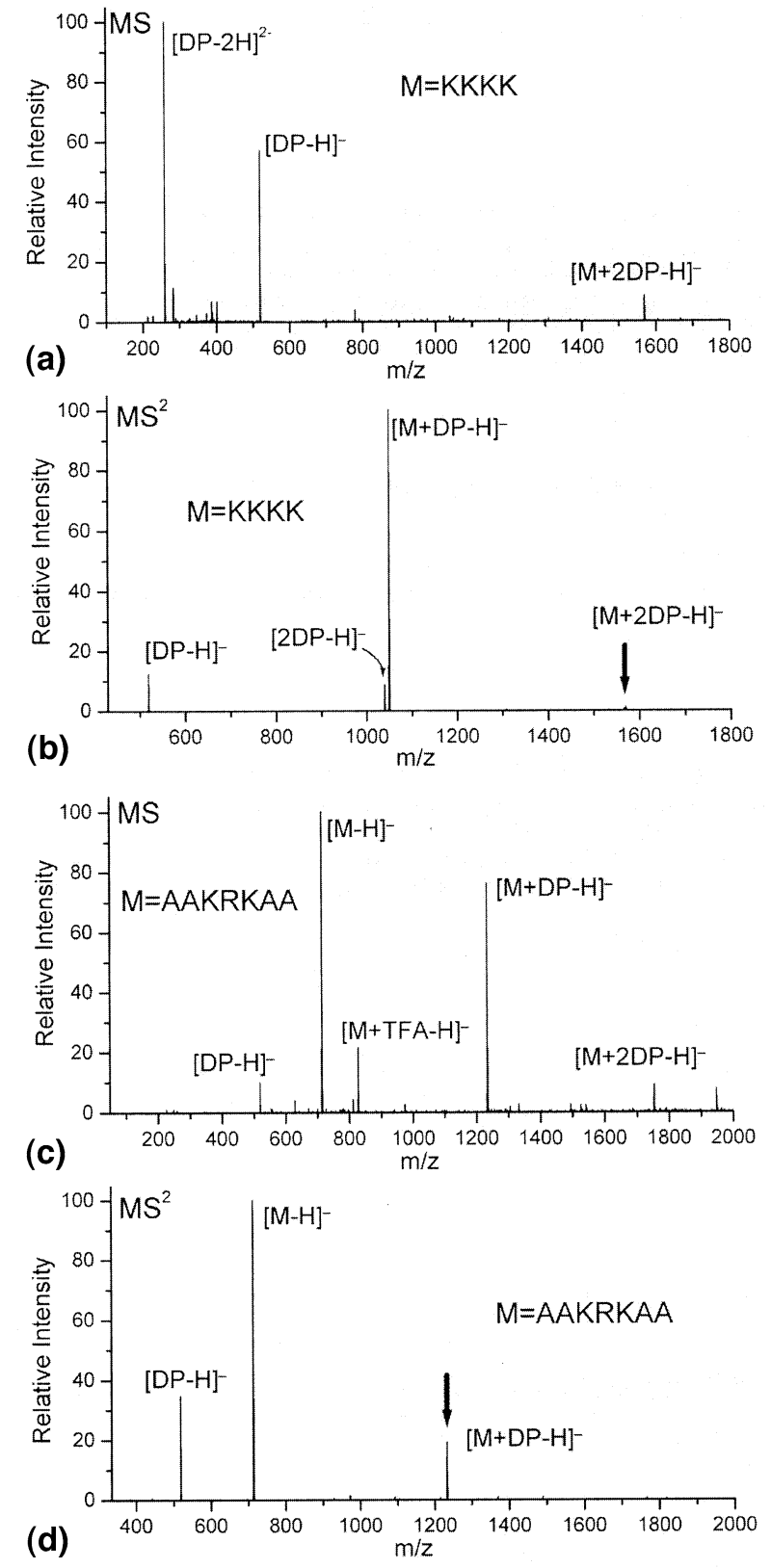

Figure 7. (a) Tetralysine forms a double anionic adduct with DP in low abundance. (b) Collisional activation leads to loss of DP and tetralysine, in addition to generating a DP dimer. (c) Complex formation between AAKRKAA and DP in anionic mode ESI. (d) Collisional activation yields the deprotonated peptide. Bold downward arrows indicate peaks being subjected to collisional activation.

applications in experiments where the presence of arginine in small peptides is of interest. For example, addition of DP would be an excellent method for the selective recognition of the arginine fragments from a tryptic digest. This approach is particularly appealing with DP because the adducts are abundant anionic species and interference from other peptides in a complex mixture will be suppressed. In other words, it may be possible to pick out only the arginine fragments from a tryptic digest by simply adding DP and looking for the most abundant anionic adduct peaks. Furthermore, DP provides an interesting scaffold for the addition of functional groups that would be capable of mediating peptide chemistry in the gas phase, similar to the molecular mousetraps based on 18C6 [5].

\section{Acknowledgments}

The authors gratefully acknowledge funding provided by NSF (grant CHE-9727566), the Beckman Institute Foundation, and the Material and Process Simulation Center for generously provided computational resources.

\section{References}

1. Graves, P. R.; Haystead, T. A. J. Molecular Biologist's Guide to Proteomics. Microbiol. Mol. Biol. Rev. 2002, 66, 40-63.

2. Aebersold, R.; Mann, M. Mass Spectrometry-Based Proteomics. Nature 2003, 422(6928), 198-207.

3. Zubarev, R. A.; Kelleher, N. L.; McLafferty, F. W. Electron Capture Dissociation of Multiply Charged Protein Cations. A Nonergodic Process. J. Am. Chem. Soc. 1998, 120, 3265-3266.

4. Julian, R. R.; Beauchamp, J. L. Site Specific Sequestering and Stabilization of Charge in Peptides by Supramolecular Adduct Formation with 18-Crown-6 Ether by way of Electrospray Ionization. Int. J. Mass. Spectrom. 2001, 210, 613-623.

5. (a) Julian, R. R.; May, J. A.; Stoltz, B. M.; Beauchamp, J. L. Molecular Mousetraps: Gas Phase Studies of the Covalent Coupling of Non-Covalent Complexes Initiated by Reactive Carbenes Formed by Controlled Activation of Diazo Precursors. Angew. Chem. Int. Ed. 2003, 42(9), 1012-1015. (b) Julian, R. R.; May, J. A.; Stoltz, B. M.; Beauchamp, J. L. Biomimetic Approaches to Gas Phase Peptide Chemistry: Combining Selective Binding Motifs with Reactive Carbene Precursors to Form Molecular Mousetraps. Int. J. Mass Spectrom. 2003, 228(2/ 3), 851-864.

6. Julian, R. R.; Akin, M.; May, J. A.; Stoltz, B. M.; Beauchamp, J. L. Molecular Recognition of Arginine in Small Peptides by Supramolecular Complexation with Dibenzo-30-Crown-10 Ether. Int. J. Mass Spectrom. 2002, 220, 87-96.

7. Bell, T. W.; Khasanov, A. B.; Drew, M. G. B.; Filikov, A.; James, T. L. A Small-Molecule Guanidinium Receptor: The Arginine Cork. Angew. Chem. Int. Ed. 1999, 38, 2543.

8. (a) Rensing, S.; Arendt, A.; Springer, A.; Grawe, T.; Schrader, T. Optimization of a Synthetic Arginine Receptor. Systematic Tuning of Noncovalent Interactions. J. Org. Chem. 2001, 66, 5814. (b) Schrader, T. H. Strong Binding of Arylguanidinium Ions by Benzylic Bisphosphonates-Evidence for $\pi$ Cation and $\pi-\pi$ Interactions. Tetr. Lett. 1998, 39, 517-520.

9. (a) Friess, S. D.; Zenobi, R. Protein Structure Information from Mass Spectrometry? Selective Titration of Arginine Residues by Sulfonates. J. Am. Soc. Mass Spectrom. 2001, 12, 810-818. (b) Friess, S. D.; Daniel, J. M.; Hartmann, R.; Zenobi, R. Mass Spectrometric Noncovalent Probing of Amino Acids in Peptides and Proteins. Int. J. Mass Spectrom. 2002, 219, 269-281.

10. Leitner, A.; Linder, W. Probing of Arginine Residues in Peptides and Proteins Using Selective Tagging and Electrospray Ionization Mass Spectrometry. J. Mass Spectrom. 2003, 38, 891-899.

11. (a) Schalley, C. A. Molecular Recognition and Supramolecular Chemistry in the Gas Phase. Mass Spectrom. Rev. 2001, 20, 253-309. (b) Smith, R. D.; Bruce, J. E.; Wu, Q. Y.; Lei, Q. P. New Mass Spectrometric Methods for the Study of Noncovalent Associations of Biopolymers. Chem. Soc. Rev. 1997, 26, 191-202. (c) Veenstra, T. D. Electrospray Ionization Mass Spectrometry 
in the Study of Biomolecular Noncovalent Interactions. Biophys. Chem. 1999, 79, 63-79. (d) Loo, J. A. Electrospray Ionization Mass Spectrometry: A Technology for Studying Noncovalent Macromolecular Complexes. Int. J. Mass Spectrom. 2000, 200, 175-186.

12. (a) Schwartz, B. L.; Light-Wahl, K. J.; Smith, R. D. Observation of Noncovalent Complexes to the Avidin Tetramer by Electrospray-Ionization Mass-Spectrometry. J. Am. Soc. Mass Spectrom. 1994, 5, 201-204. (b) Nemirovskiy, O. V.; Ramanathan, R.; Gross, M. L. Investigation of Calcium-Induced, Noncovalent Association of Calmodulin with Melittin by Electrospray Ionization Mass Spectrometry. J. Am. Soc. Mass Spectrom. 1997, 8, 809-812. (c) Brodbelt, J. S. Probing Molecular Recognition by Mass Spectrometry. J. Mass Spectrom. 2000, 200, 57-69. (d) Eckart, K.; Spiess, J. Electrospray-Ionization Mass-Spectro- metry of Biotin Binding to Streptavidin. J. Am. Soc. Mass Spectrom. 1995, 6, 912-919.

13. Gokel, G. W., Ed.; Comprehensive Supramolecular Chemistry, Vol. I; Pergamon/Elsevier: Oxford, 1996.

14. Wyttenbach, T.; von Helden, G.; Bowers, M. T. Gas-Phase Conformation of Biological Molecules: Bradykinin. J. Am. Chem. Soc. 1996, 118, 8355-8364.

15. Hunter, E. P.; Lias, S. G. Proton Affinity Evaluation. In NIST Chemistry WebBook, NIST Standard Reference Database Number 69; Mallard, W. G.; Linstrom, P. J., Eds.; National Institute of Standards and Technology: Gaithersburg MD, 2000 (http:// webbook.nist.govhttp:/ / webbook.nist.gov).

16. O'Hair, R. A. J.; Bowie, J. H.; Gronert, S. Gas-Phase Acidities of the $\alpha$-Amino-Acids. Int. J. Mass Spectrom. Ion Processes 1992, $117,23-36$. 